\title{
Proceedings of the summer meeting of the British Neuropsychiatric Association, Manchester Conference Centre, 25-27 September 1994
}

The topics of the meeting were recent developments in dementia and epilepsy.

Dr C Wischik (Cambridge) was the keynote speaker in the dementia session with his talk on molecular substrates of clinical dementia in Alzheimer's disease: pathological mechanisms and therapeutic strategies. He reviewed recent research on the role of tau protein, a microtubule stabiliser that plays an important part in the transport of synaptic constituents. Whereas in normal controls tau protein is mostly in a free soluble form, it is largely polymerised in Alzheimer's disease. These alterations, which may be due to abnormal phosphorylation or truncation, result in breakdown of axonal function and deposition of paired helical filaments. Wischik's own work has shown that the severity of cognitive impairment in Alzheimer's disease correlates with the deposition of paired helical filaments and inversely with the amount of soluble tau protein present in medial temporal lobe structures. Deposition of paired helical filaments is also related to cellular destruction. Abnormalities in tau protein do not seem to be related to the increased prevalence of the Apo-E4 allele, also present in cortical Lewy body disease, and may be pathognomonic to Alzheimer's disease. On the other hand, high aluminium concentrations may trigger these abnormalities. The understanding of these changes and possible prevention will open new avenues for treatment.

Professor A Burns (Manchester) gave an overview of new developments in Alzheimer's disease. Genetic advances have led to the recognition of the locus of the amyloid precursor protein gene in chromosome 21 , which, together with the recent evidence of linkage to chromosome 14 in some families, probably accounts for most early onset familial cases. Late onset cases have been linked to abnormalities in chromosome 19 associated with the presence of the Apo-E4 allele. Diagnosis has also been improved by the use of structural and functional neuroimaging. The use of cholinergic agents in treatment (release enhancers, tetrahydroaminoacridine) is still under investigation.

Professor F Owen (Manchester) discussed molecular biological aspects of dementia. Recent research, including his own, has shown that the possession of the E4 isoform of the Apo-E allele is a major risk factor in the development of Alzheimer's disease and cortical Lewy body disease, but not of lobar atrophies. It has also been suggested that the E2 isoform, and to a lesser extent the $\mathrm{E} 3$ isoform, have a neuroprotective role and that they promote longevity. This neuroprotection, which may also be present in Down's syndrome, results from their ability to bind to tau protein, reducing the rate of phosphorylation.

Dr J Snowden (Manchester) described semantic processing in dementia. A loss of ability to understand the meaning of words is a salient feature of dementia, usually as part of a widespread language disorder that includes disturbances in phonology, syntax, and word retrieval. Isolated semantic deficits can, however, appear in association with temporal lobe atrophy in patients with lobar atrophy of nonAlzheimer type. In these patients the semantic disorder extends to the meaning of faces, objects, tastes, odours, and non-verbal sounds in the presence of other preserved cognitive abilities. These rare cases support the theoretical distinction between semantic and episodic memory and are important in teasing out the various processes involved in language function.

Dr G Goodwin (Edinburgh) in his talk on functional brain imaging and the actions of antidementia drugs discussed the uses of SPECT in studying the neural substrates subserving memory and the way in which they are influenced by cholinergic stimulation. Work by his group with the SPECT ligand ${ }^{99 m} \mathrm{Tc}$ exametazime has shown activation of the dorsolateral frontal cortex and adjacent anterior cingulate in normal controls, but not in patients with Alzheimer's disease, during performance of a recognition memory task. On the other hand, increased regional perfusion and metabolism in these regions were found in patients with Alzheimer's disease after treatment with velnacrine maleate, a cholinesterase inhibitor. These effects were more pronounced in patients with severe cognitive impairment, suggesting cholinergic supersensitivity.

Dr D Crauford (Manchester) reviewed recent developments in the genetics of Huntington's disease. The mapping of the gene for Huntington's disease in 1993 to the area $4 p 16,3$ of chromosome 4 is an important landmark. The development of the disease and age of onset seem to be related to the increase in size of the trinucleotide repeat CAG. The size of this repeat in Huntington's disease varies between 42 and 100, compared with 11 to 34 for normal subjects. Dr Crauford's work, in accordance with that of other laboratories, has found little overlap in the number of CAG repeats in normal 
subjects and patients with Huntington's disease and has highlighted the greater number of repeats in those with onset before the age of 25 (mean 61 repeats) compared with those with an age of onset over 40 (mean 43 repeats). Paternal transmission seems to be associated with an increase in the number of repeats in the affected child, whereas this is not the case in maternal transmission. Paternal transmission may account for the so called "sporadic" patients whose fathers had an intermediate range of repeats (30-38). Expanded CAG repeats, albeit in other chromosomes, have also been reported in other disorders such as spinobulbar muscular atrophy and spinocerebellar ataxia type I.

The session ended with the following scientific papers and video case presentations: apolipoprotein $\mathrm{E}$ (Dr S Lovestone); selective damage to CA hippocampal fields is sufficient to produce severe amnesia (Dr L Kartsounis); problem solving in schizophrenia: a specific deficit in planning ability ( $\mathrm{Dr} \mathrm{R}$ Morris); catatonic and extrapyramidal motor disorder in autism (Dr P McKenna); an unusual form of presenile dementia ( $\mathrm{Dr} M$ Thomas); an unusual motor disorder in a patient with generalised epilepsy (Dr S Simpson); movement disorder in a four year old child with developmental delay and self injurious behaviour (Drs $S$ Warren and $J$ Bernal); periodic hypersomnia of early onset in a boy with Asperger's syndrome (Dr M Prendergast); antisocial behaviour in Asperger's syndrome (Dr K O'Driscoll).

Dr S Shorvon (London) was the key speaker in the epilepsy session with his lecture on advances in the understanding of the structural basis of epilepsy: the contribution of MRI. He argued that the advent of MRI is as important an advance in epilepsy as the introduction of EEG in the 1930s. Magnetic resonance imaging has been particularly useful in imaging the hippocampus to detect and measure the extent of sclerosis and atrophy, aiding greatly in the presurgical assessment of epilepsy. It has also made it possible to detect small foreign tissue lesions (hamartomas, cavernous haemangiomas, and small gliomas) and cortical dysplasias, which seem to be very important in the causation of epilepsy and are increasingly detected and characterised. Magnetic resonance imaging is also used in stereotactic planning and depth electrode placement and allows, using coregistration of presurgical and postsurgical images, outcome to be related to site and extent of resection. He concluded his talk by discussing new technical advances in MRI such as postprocessing techniques and new methods of acquisition and quantification of data, which hold great promise.

This theme was continued by Dr David Fish (London) who discussed cortical dysgenesis, now recognised as one of the most important causes of epilepsy. Errors in neuronal migration and allied developmental processes result in gyral abnormalities, heterotopias, focal dysplasias, microdysgenesis, and proliferative lesions. Dual pathology is being increasingly recognised and in his own series of 100 patients with hippocampal sclerosis identified on MRI, distant areas of cortical dysplasia were found in 15. Patients with epilepsy associated with cortical dysgenesis tend to present in the first two decades of life and, except in those with gross abnormalities, their IQ is within the normal range. In patients with intractable epilepsy who were studied by Fish this pathology was present in $24 \%$ and the possibility has recently been raised that other factors may be necessary to trigger epilepsy in these patients. A variety of insults occurring during the crucial period of cortical development could account for these abnormalities, but in most cases the cause remains unknown.

The classification of epileptic syndromes in childhood was dealt with by Dr H Cross (London). Recent classifications have tended to use syndromes rather than specific types of seizures. These syndromes are defined by clinical and EEG characteristics, with an increasing contribution from imaging. Epilepsy syndromes are age related and divided into "benign", usually genetically determined with good cognitive development and seizure control, and "malignant", accompanied by cognitive deficits and intractable seizures. This dichotomy is not always clear, however, and considerable overlap exists between syndromes. The better understanding of cortical dysgenesis, improved imaging, and new anticonvulsant drugs will assist in the management of these patients.

Dr S Brown (Manchester) discussed cognitive decline and dementia in epilepsy. The reasons for this decline are varied and may include trauma, drugs, interictal epileptiform activity, and coexisting degenerative disease. $\mathrm{He}$ presented the neuropsychological findings in a small series of chronic epileptic patients who showed intellectual decline years after the onset of epilepsy and in whom frontotemporal skills seem to be preferentially affected. Reduction of cerebral blood flow in the frontotemporal regions demonstrated by SPECT supported these clinical findings.

Professor M Trimble (London) dealt with psychosis in epilepsy. He focused attention on patients who develop psychosis after temporal lobectomy. The incidence of psychosis is unknown, but it has been reported on some occasions after a free interval. Anticonvulsants can also cause psychosis and among the new drugs lamotrigine and vigabatrin are of particular interest. In his own series 14 out of 210 patients treated with vigabatrin developed psychosis, nine for the first time. From examination of pooled data from other groups it seems that in half of the patients a latent period elapsed between the initiation of drug treatment, and the onset of psychotic symptoms. There was a tendency for the psychosis to be associated with higher doses and to be commoner in those who had a previous psychiatric history and more severe epilepsy. Intriguingly, psychosis may be associated with good epilepsy control and it seems possible 
that dopamine release may mediate the psychotic symptoms.

Finally, Professor A Richens (Cardiff) provided an overview of new antiepileptic drugs, focusing on their indications and adverse psychiatric effects.

The session ended with the following papers and video case presentations: mesial temporal sclerosis and febrile convulsions (Professor D Taylor); validity and reliability of the BEA epilepsy screening questionnaire (Dr D Jadresic); effects of new antiepileptic drugs in individuals with learning difficulties; results of an open add on study of lamotrigine (Dr Staufenberg); non-epileptic attack disorder (Dr B Scheepers).

At the Annual General Meeting it was announced that Professor C Frith, Professor of Psychology at the Institute of Neurology, will join the committee to replace Dr F Huppert.
Professor A Lishman, Honorary President, announced that there had been many excellent entries for the first BNPA prize and that the Association had taken the decision to award two prizes on this occasion given the exceptional quality of two of the entries. The winners were Dr E Bullmore for his paper entitled computerised brain tissue classification of magnetic resonance images: a combined statistical and morphological approach to the problem of partial volume artifact and Dr J Powell for her work on poor motivation in patients with organic brain injury: evidence for underlying dopamine dysfunction and effective pharmacological intervention. Both winners will present papers at the 1995 summer meeting in Cambridge.

M A RON

The National Hospital for Neurology and Neurosurgery, Queen Square, London, 